\title{
ISOLATION OF CELLULOSE DERIVED FROM ORANGE PEEL AND ITS APPLICATION IN BIODEGRADABLE FILMS
}

\author{
VANESSA NG WEN YI, NG CHOO HUEY, ${ }^{* *}$ TEOH YI PENG, ${ }^{*}$ \\ OOI ZHONG XIAN*** and SHUIT SIEW HOONG ${ }^{* * *}$ \\ "Department of Petrochemical Engineering, Faculty of Engineering and Green Technology, \\ Universiti Tunku Abdul Rahman, Jalan Universiti, Bandar Barat, \\ 31900 Kampar, Perak, Malaysia \\ ** Department of Chemical Science, Faculty of Science, Universiti Tunku Abdul Rahman, \\ Jalan Universiti, Bandar Barat, 31900 Kampar, Perak, Malaysia \\ ${ }^{* * *}$ Department of Chemical Engineering, Lee Kong Chian Faculty of Engineering and Science, Universiti \\ Tunku Abdul Rahman, Sungai Long Campus, Jalan Sungai Long, Cheras, \\ 43000 Kajang, Selangor, Malaysia \\ \Corresponding author: Ooi Zhong Xian,ooizx@utar.edu.my
}

Received October 23, 2020

In this study, cellulose was extracted and isolated from orange peel (OP) via alkaline treatment, followed by a bleaching process. The orange peel derived cellulose (OPDC) was characterized and compared with microcrystalline cellulose (MCC). FTIR characterization confirmed the absorption peaks of cellulose for both OPDC and MCC. From the DSC analysis, it was found that MCC possessed higher thermal stability than OPDC. In addition, the results of FESEM and particle size analysis revealed the micro-size dimension of OPDC after the pretreatments. The crystallinity index (CrI) of OPDC (80.14\%) was found to be significantly higher than that of untreated OP (23.54\%). After that, biodegradable blended films with different weight ratio were prepared using OPDC and MCC with polyvinyl alcohol (PVA) by the solution casting method. According to the FTIR spectra, the shift of the characteristic absorption band and the change of the band intensity observed in the spectra of the PVA/OPDC blended films were similar to those of the PVA/MCC blended films. This confirmed the development of new inter- and intramolecular hydrogen bonds and changes in the conformation between PVA and cellulose. The tensile strength and elongation at break of both PVA/OPDC and PVA/MCC blended films decreased when the cellulose content increased in the PVA matrix. However, the elastic modulus of both films was enhanced when the amount of cellulose increased. By comparison, the PVA/OPDC blended film presented higher tensile strength and elastic modulus than the PVA/MCC blended film. Based on the natural weathering tests, the degradability of blended films increased with a rising amount of cellulose after exposure to degradation tests. The biodegradability of blended films was confirmed by the tiny black spots observed on their surface, which reflected the growth of microorganisms. It was further proven by the morphology studies performed on the surface of the films using FESEM.

Keywords: orange peel (OP), orange peel derived cellulose (OPDC), biodegradable blended film, polyvinyl alcohol (PVA)

\section{INTRODUCTION}

Citrus fruits, such as orange, grapefruit and lemon, are one of the most consumed and wellknown types of fruits around the world, due to their beneficial nutritional properties. They also contain many phytochemicals, which provide protection against gastric, oesophageal and colorectal diseases. ${ }^{1}$

Citrus fruits, like all plant materials, including natural lignocellulosic materials, consist of three main organic compounds, namely cellulose, hemicelluloses and lignin. Cellulose, which is primarily found in plant cells, is the most abundant renewable resource on earth. It is a kind of biodegradable biopolymer that can be decomposed by microorganisms available in soil naturally into carbon dioxide and water. In addition, it is a large-molecule polymer, composed of repeating units of D-glucose units 
joined by $\beta-1,4-$ glycosidic bonds. A hydrogen bond is the main chemical bond that builds the large network of the crystalline structure of cellulose. However, the non-crystalline phases are also found in cellulose. This amorphous region is caused by the hydroxyl groups in the glucose unit. $^{2}$

Natural cellulose fibres can be isolated from waste plant biomass and cellulose derivatives via chemical modification of cellulose. Cellulose derivatives are strong, biocompatible and can be recycled or reproduced. ${ }^{3}$ Cellulose and its derivatives have a wide range of applications, such as paper products, films from cellulose acetate, composites, upholstery, cotton and textile materials. Cellulose films, produced from cellulose fibres, were the first transparent packaging films used for snack foods, biscuits, as well as for adhesive tapes. Cellophane is a common name for a cellulose film, which is a renewable resource and a biodegradable polymer, with good clarity, glitter and eye-catching colours. Moreover, the dead fold properties of cellophane are the main factor that allows its use in twist wrapping of sweets and chocolates. ${ }^{4}$

Plastics have become one of the most important materials in human lives. Plastic materials include polyethylene (PE), polyethylene terephthalate (PET), polyvinyl chloride (PVC), polyamide (PA) also known as nylon, polystyrene (PS), polyester (PES), and polypropylene (PP). However, the production of plastics from petroleum has raised the issue of environmental pollution because of their non-biodegradable properties. ${ }^{5}$ According to Eich et al., ${ }^{6}$ plastic is considered stable as it requires several hundred years of degradation time, depending on the types of plastics. Most of the plastic types are bio-inert and non-biodegradable because of the hydrophobic properties and high molecular weight of the synthetic polymers that prevent phagocytosis. Consequently, the overall decomposition of microbial plastic is very low even though some microorganisms are found capable to degrade plastics.

One of the attempts to overcome the disposal of plastic waste is to produce environmentally friendly biodegradable plastics. There are many kinds of biodegradable polymers, such as polylactic acid (PLA) and polycaprolactone (PCL). Of them, polyvinyl alcohol (PVA) is the most promising and widely used polymer. PVA is a water-soluble synthetic polymer, and one of the most promising materials among the biodegradable plastic sources due to its biodegradability, biocompatibility, high tensile strength, high-modulus and easy processing. ${ }^{7}$ In addition, the thermoplasticity of PVA makes the solution casting method possible. ${ }^{8}$ Nevertheless, pure PVA is often insufficient for its use because of its brittleness, flammability, poor thermal stability and solvent resistance. Thus, it requires reinforcement to improve its properties. ${ }^{7}$

Eco-sustainable composites can be obtained by mixing PVA with natural cellulose fibres. Cellulose fibres were reported to be compatible with PVA due to the hydrophilic nature of hydroxyl groups present in cellulose, establishing excellent composite properties and good performance. ${ }^{9}$ Based on the data reported in the literature, PVA was blended with several natural biopolymers to enhance biodegradability and reduce production costs. The most widely used and well-known biodegradable polymer in the packaging and agriculture industries is the PVA/starch blended film. Moreover, other reinforcement materials, such as the lignocellulosic compounds banana frond flour ${ }^{10}$ (BFF) and kenaf/soy protein, ${ }^{11}$ have been investigated.

\section{EXPERIMENTAL}

\section{Materials and chemicals}

Valencia oranges were purchased from Northridge International Sdn. Bhd., located in Batu Caves, Kuala Lumpur, Malaysia, and the orange peels were collected and used as raw material in this research. The chemicals used include: ethanol and microcrystalline cellulose purchased from Sime Scientific, toluene and glycerol purchased from Friendemann Schmidt, sodium hydroxide purchased from Merck (Germany), sodium chlorite $\left(\mathrm{NaClO}_{2}, 80 \%\right)$ purchased from Acros Organics (Spain), acetic acid glacial purchased from Bendosen, and PVA purchased from Sigma Aldrich (USA).

\section{Preparation and maceration of orange peels}

The collected OP was cut into smaller pieces, washed with water and oven-dried at $80{ }^{\circ} \mathrm{C}$ overnight. The dried OP was then ground into powder. Maceration of the ground OP in an ethanol/toluene solution was done for 3 days, whereby the ratio of fibre to solution was set to $1: 15$. The extracted orange oil was removed, and the macerated OP was rinsed with distilled water. It was then centrifuged at $3500 \mathrm{rpm}$ for 10 minutes to remove the solvent completely and the sample was dried in an oven at $50{ }^{\circ} \mathrm{C}$ overnight.

\section{Extraction of cellulose}

Alkaline treatment was performed by using $5 \%$ 
(w/v) sodium hydroxide $(\mathrm{NaOH})$, with 1:15 ratio, heated at about $80{ }^{\circ} \mathrm{C}$ for 3 hours. The cellulose was centrifuged at $3500 \mathrm{rpm}$ for 10 minutes and washed with distilled water for several times until a neutral $\mathrm{pH}$ was obtained. The fibres were then subjected to bleaching with $1.7 \%(\mathrm{w} / \mathrm{v}) \mathrm{NaClO}_{2}$, at a fibre to solvent ratio of $1: 15$. The mixture was acidified to $\mathrm{pH}$ 4 , with the addition of glacial acetic acid, and heated at about $80{ }^{\circ} \mathrm{C}$ for 2 hours. It was then cooled, centrifuged at $3500 \mathrm{rpm}$ for 10 minutes and washed with distilled water until white cellulose was obtained. It was then dried in the oven at $50{ }^{\circ} \mathrm{C}$ overnight. The percentage yield of OPDC was calculated by Equation (1):

Yleld $=\frac{M_{2}(g)}{M_{1}(g)} \times 100 \%$

where $M_{1}$ is the mass of macerated OP used, while $M_{2}$ is the mass of OPDC produced.

\section{Preparation of PVA blended films}

A total of $2 \%(\mathrm{w} / \mathrm{v})$ of varying composition of PVA/OPDC and PVA/MCC blended films were prepared. In a beaker, the cellulose was dispersed in distilled water and stirred at $35{ }^{\circ} \mathrm{C}$ for 30 minutes. Glycerol was then added into the suspension and continuously stirred for another 30 minutes. At the same time, the PVA solution was stirred at about $90{ }^{\circ} \mathrm{C}$ for an hour in a separate beaker. Then, the cellulose suspension was added into the PVA solution and stirred for 1 hour. When OPDC or MCC was dispersed in water, cellulose formed a very stable white cloudy suspension due to the strong hydrophilic characteristics of cellulose. When PVA and cellulose were mixed together, a more viscous suspension was formed due to the high affinity of PVA to bind with cellulose through hydrogen bonding. After that, the PVA/cellulose solution was poured onto a Petri dish to cast the blended film. The blended film was air dried overnight and further dried in the oven at $50{ }^{\circ} \mathrm{C}$ for 2 days. The same procedures were repeated to cast different compositions of PVA/cellulose blended films with the ratios: $2.0: 0,1.5: 0.5,1.0: 1.0,0.5: 1.5$ and $0: 2.0$. It was observed that the unfilled PVA film had high transparency, while the transparency of the PVA/OPDC and PVA/MCC blended films was diminished. On the other hand, a white colour film was formed when the amount of cellulose added was increased. The physical appearance of the PVA/OPDC and PVA/CMC blended films with different compositions is shown in Figure 1. Lastly, the films were kept in a desiccator prior to further analysis.

\section{Fourier transform infrared (FTIR) spectroscopy}

The FTIR spectra for OP, OPDC, microcrystalline cellulose (MCC) and the blended films were obtained by using an FTIR spectrophotometer (brand: Perkin Elmer, model: Spectrum Two). An appropriate amount of sample was placed on the diamond cell. The pressure controller was then screwed down until the pressure gauge on the software program showed the optimum level of pressure between 60 to $80 \%$. The FTIR spectra were analysed in the wavenumber range from 400 to $4000 \mathrm{~cm}^{-1}$, with a resolution of $4 \mathrm{~cm}^{-1}$ for 32 scans.

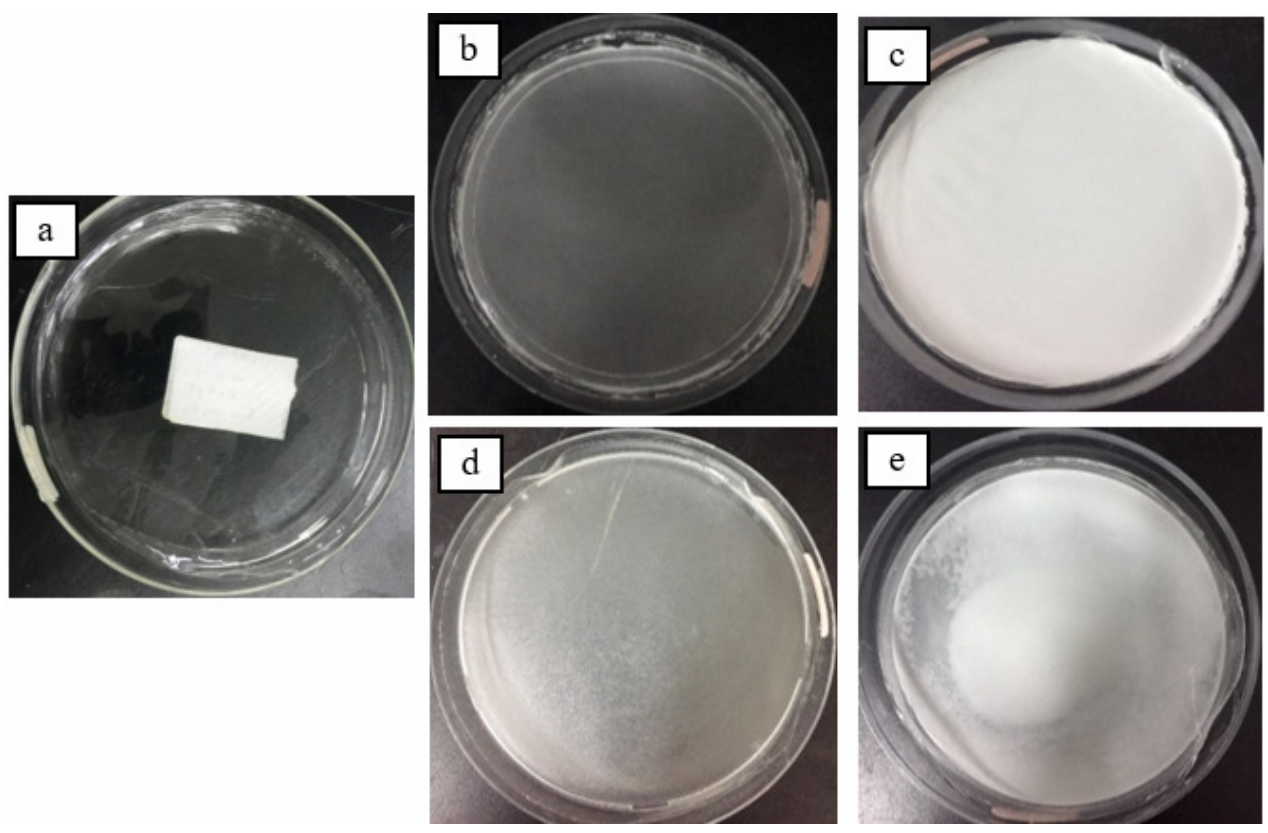

Figure 1: Physical appearance of (a) unfilled PVA, (b) PVA/OPDC 1.5:0.5 blended film, (c) PVA/OPDC 1.0:1.0 blended film, (d) PVA/MCC 1.5:0.5 blended film, (e) PVA/MCC 1.0:1.0 blended film 


\section{Field emission scanning electron microscopy (FESEM)}

A JEOL Japan SEM, JSM-6701F, was used to study the surface morphology and microstructure of the fibres and degraded films. The sample was deposited on the copper stub with the aid of carbon tape and was left to dry in the oven at $50{ }^{\circ} \mathrm{C}$ for 20 minutes. The sample was sputter coated with a thin layer of platinum (thickness $15 \mathrm{~nm}$, density 21.45 $\mathrm{g} / \mathrm{cm}^{3}$ ) by using a sputtering machine (brand: JEOL, model: JFC-1600). This step was performed to avoid electrostatic charging and poor resolution during analysis. The images of the morphology of samples can be observed with magnification of $\times 500, \times 1000$, $\times 2000, \times 10000, \times 20000$ and $\times 50000$ at a $4.0 \mathrm{kV}$ accelerating rate.

\section{Particle size analysis (PSA)}

A particle size analyser (brand: Malvern, model: Masterizer (Hydro $2000 \mathrm{MU}$ )) was used to determine the size of OPDC and MCC. Firstly, a refractometer (brand: Lauda; model: Alpha RA $\delta$ ) was used to determine the refractive index of both OPDC and MCC. During analysis, distilled water was used as suspension medium for dispersion of cellulose particles. Then, the probe of the particle size analyser was immersed into the suspension for analysis.

\section{Differential scanning calorimetry (DSC)}

DSC (brand: Mettler Toledo; model: DSC1, with an automatic voltage regulator) was conducted to determine the thermal properties of OPDC and MCC. About 2-3 mg of sample was weighed and sealed into an aluminium crucible. An empty aluminium crucible was used as reference cell. DSC analysis was carried out at a heating rate of $10{ }^{\circ} \mathrm{C} / \mathrm{min}$, from $25{ }^{\circ} \mathrm{C}$ to 280 ${ }^{\circ} \mathrm{C}$, with two heating and one cooling scan in nitrogen atmosphere.

\section{X-ray diffraction (XRD)}

OP, OPDC and MCC were analysed using XRD to determine the crystallinity of cellulose. A Shimadzu XRD 6000 X-ray diffractometer, operating at $40 \mathrm{kV}$ and $30 \mathrm{~mA}$, was used to obtain the diffraction profile at $2{ }^{\circ} \mathrm{C}$ per min. Samples were scanned using a monochromatic $\mathrm{Cu}-\mathrm{K} \alpha$ radiation source $(\lambda=1.5406$ $\AA)$, in the step-scan mode, with a $2 \theta$ angle ranging from $5^{\circ}$ to $70^{\circ}$. The crystallinity index, $\mathrm{CrI}$ or the degree of crystallinity, was determined by Segal's method, using Equation (2), as outlined by Chieng et $a l .:^{12}$

$\% C r l=\frac{I_{002}-I_{a m}}{l_{002}} \times 100 \%$

where $I_{002}$ provides the maximum peak intensity value for the crystalline cellulose at about $2 \theta=22.0^{\circ}$ to $24.0^{\circ}$ and $I_{\mathrm{am}}$ gives the peak intensity of diffraction of the amorphous region at about $2 \theta=18.0^{\circ}$ for cellulose type I. ${ }^{13,14}$

\section{Tensile properties}

The tensile strength and elongation at break of the composite films was measured on a Tinius Olsen H10KS tensile tester, by applying a $450 \mathrm{~N}$ load on the film, with a speed of $10 \mathrm{~mm} / \mathrm{min}$. A dumbbell cutter was used to cut the film with a gauge length of $26 \mathrm{~mm}$ and width of $3 \mathrm{~mm}$. A thickness gauge (brand: Mitutoyo) was used to measure the film thickness by taking the reading at three different points to obtain the average value. An average result was obtained by measuring five specimens for each composite film.

\section{Natural weathering test}

The aim of the natural weathering test was to determine the biodegradability of the film. The film was cut into a smaller rectangular shape, with approximately the same dimensions for each formulation. The film was dried overnight and weighed before exposure to the environment. The natural weathering test was carried out on the rooftop of Block E, University Tunku Abdul Rahman (UTAR), Kampar Campus, Perak. The specimens were attached to the inclined weathering rack. The weathering rack was oriented at an angle of $45^{\circ}$ and was positioned in an open area that was free from overshadowing by other objects. This test was carried out for 30 days and the weight loss of the film was measured weekly using a weighing balance. Equation (3) shows the calculation for percentage of film degradation:

Degradation $\%=\frac{W_{1}(g)-W_{2}(g)}{W_{1}(g)} \times 100 \%$

where $W_{1}$ is the initial weight of the film and $W_{2}$ is the final weight of the film after degradation.

\section{RESULTS AND DISCUSSION Percentage yield of OPDC}

The average percentage yield of cellulose from orange peel used after the alkaline and bleaching treatment was $20.28 \%$. According to Ilyas, Sapuan and Ishak, ${ }^{15}$ the weight loss was an indication of the removal of hemicelluloses, lignin, pectin, fat, impurities, wax and oils from $\mathrm{OP}$, contributing to the very low percentage yield of OPDC. The high weight loss may also be caused to the three runs of alkaline treatment to obtain a higher purity of OPDC.

\section{FTIR spectroscopy of OP, OPDC and MCC}

FTIR spectroscopy was used to determine the presence or absence of functional groups before and after the treatment, as well as to identify changes in the intensity of peaks. Figure 2 shows the FTIR spectra of OP, OPDC and MCC. The FTIR spectra of OPDC were compared with the reference sample, MCC. The appearance of peaks on the spectra of OPDC corresponded to the bands of MCC. Meanwhile differences in the 
absorption peak intensities and the presence of new peaks were observed in the spectra of OP. The broad absorption bands around 3200-3300 $\mathrm{cm}^{-1}$ regions indicated the $\mathrm{O}-\mathrm{H}$ stretching vibration, whereas the absorption peaks around $1600-1640 \mathrm{~cm}^{-1}$ showed the O-H bending of absorbed water. The absorption peaks near 2900 $\mathrm{cm}^{-1}$ corresponded to the aliphatic $\mathrm{C}-\mathrm{H}$ stretching vibration of all hydrocarbon constituents in polysaccharides. ${ }^{16}$ The intensity of the $\mathrm{C}-\mathrm{H}$ stretching peak of OPDC and MCC was slightly lower than that of OP, indicating that both the lignin and hemicelluloses were removed from OP during the bleaching process. ${ }^{17}$

One distinguishable vibration peak at $1736 \mathrm{~cm}^{-}$ ${ }^{1}$ in the spectra of OP can be ascribed to the presence of the $\mathrm{C}=\mathrm{O}$ stretching vibration of the acetyl group of hemicelluloses. The disappearance of this peak in the spectra of OPDC and MCC was due to the removal of hemicelluloses after alkaline treatment. ${ }^{17,18} \mathrm{Next}$, the absorption peak at $1236 \mathrm{~cm}^{-1}$ indicated the $\mathrm{C}-$ $\mathrm{O}$ stretching of the aryl group of lignin. Such a peak was found to be absent in the spectra of OPDC and MCC. This portrayed the partial removal of lignin from the OP surface. ${ }^{17}$ The absence of the two peaks in the spectra of OPDC and MCC implied that alkaline treatment successfully removed most of the lignin and hemicelluloses found in OP. ${ }^{19}$ Furthermore, the peaks in the regions around $1160 \mathrm{~cm}^{-1}$ were assigned to $\mathrm{C}-\mathrm{O}-\mathrm{C}$ asymmetrical ring stretching. ${ }^{19,20}$ While the peaks in the regions around 1012-1039 $\mathrm{cm}^{-1}$ were contributed by the $\mathrm{C}-\mathrm{O}-\mathrm{C}$ bending of the pyranose ring in the cellulose. ${ }^{17}$ Lastly, the peaks found on both OPDC and MCC spectra at 1425-1428 $\mathrm{cm}^{-1}$ indicated $\mathrm{CH}_{2}$ symmetric bending, while the peak at $896 \mathrm{~cm}^{-1}$ were contributed by the stretching of the $\beta$-glycosidic linkages of cellulose. ${ }^{20}$

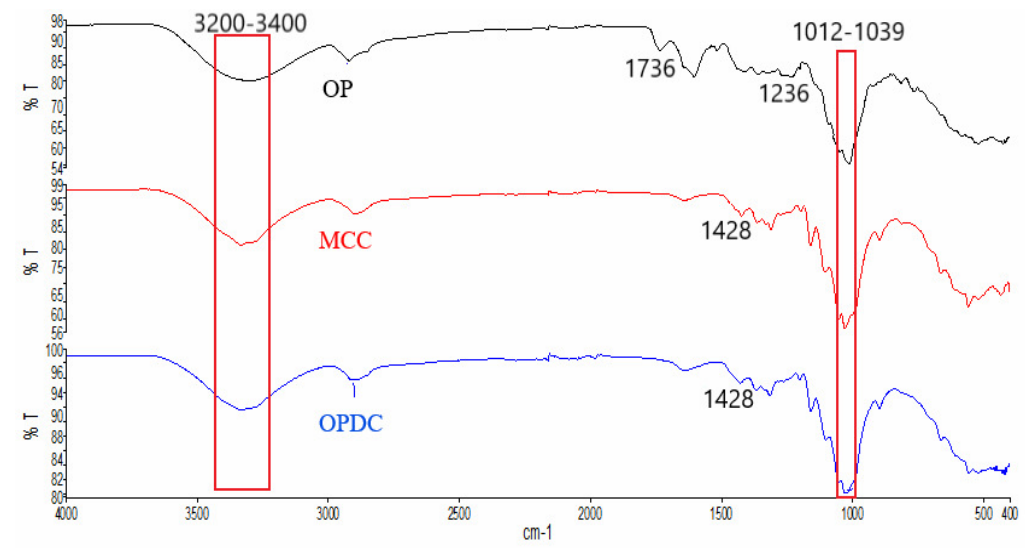

Figure 2: FTIR spectra of OP, MCC and OPDC
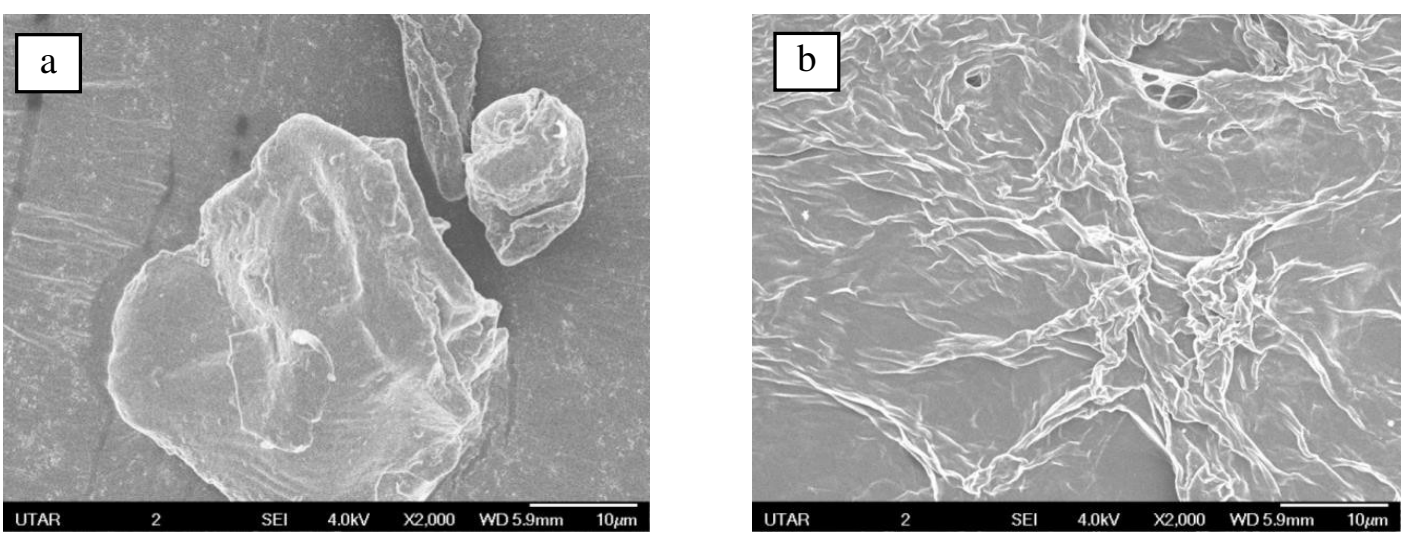

Figure 3: FESEM images of surface morphology of untreated (a) OP and (b) OPDC at magnification of $\times 2000$ 


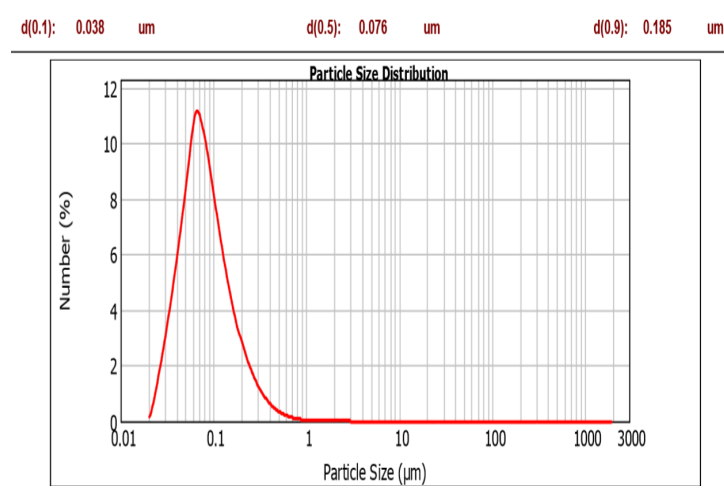

Figure 4: Particle size distribution of OPDC

\section{FESEM of OP and OPDC}

Figure 3 shows the surface morphologies of untreated OP and OPDC, respectively. The untreated $\mathrm{OP}$ in Figure 3 (a) reveals a nonuniform surface, with many non-cellulosic materials of irregular shape, such as the gummy polysaccharides of hemicelluloses, lignin, pectin and waxes, scattered over the surface. ${ }^{28}$ The surface was smooth and the orientation of cellulose microfibril aggregates was not prominent in OP. However, it can be clearly observed in OPDC, as shown in Figure 3 (b). ${ }^{29}$ In addition, the delignified OPDC, with a cleaner surface, indicated the removal of lignin and other extractives, such as waxes, oil and pectin, after the delignification process using acidified sodium chlorite. The uniform distribution of microfibrillated cellulose exhibited in Figure 3 (b) also revealed the removal of hemicelluloses from OP by the alkaline treatment. ${ }^{30}$

\section{Particle size analysis}

The refractive index of OPDC and MCC was 1.33. Figures 4 and 5 show the particle size distribution of OPDC and MCC, respectively. According to Figure 4, $11 \%$ of the total amount of OPDC particles were in the range from 0.060 to $0.091 \mu \mathrm{m}$. On the contrary, Figure 5 shows the particle size distribution of MCC, in which 13\% of the total amount of particles were found in the range from 0.069 to $0.105 \mu \mathrm{m}$. The results of the study revealed that OPDC turned to micro-size dimensions after the alkaline treatment. In addition, OPDC was found to have smaller average particle size, of $0.076 \mu \mathrm{m}$, as compared to that of MCC, of $0.088 \mu \mathrm{m}$.

\section{DSC analysis}

A DSC test was carried out for OPDC in comparison with MCC as a reference material.

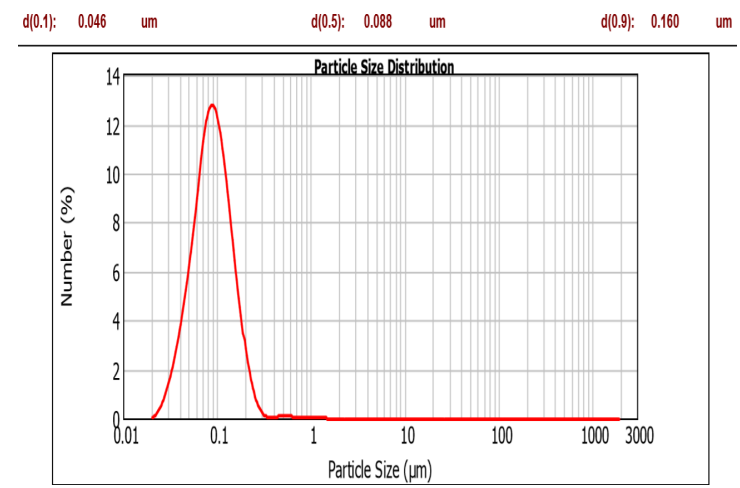

Figure 5: Particle size distribution of MCC

Figures 6 and 7 show the thermal behaviour of OPDC and MCC. By comparing the graphs, OPDC showed a very close melting temperature, $\mathrm{T}_{\mathrm{m}}$, to that of MCC due to the elimination of hemicelluloses after acid hydrolysis of the $\mathrm{MCC}^{14}$ and OPDC.

A slight peak around $125^{\circ} \mathrm{C}$ was detected in the DSC curve of MCC, which may not be a peak, but rather noise. Therefore, the peak was not regarded as an indication of crystallinity. ${ }^{33}$ Lastly, higher onset temperatures were often attributed to higher thermal stability. Hence, MCC has higher thermal stability and is more widely applicable in food stabilizers, biocomposites and pharmaceutical compounds. ${ }^{14}$

\section{XRD analysis}

XRD analysis was carried out to determine the crystallinity index, in addition to the changes in the crystallinity and amorphous region of untreated OP and OPDC after the treatment, in comparison with commercial cellulose (MCC). ${ }^{1}$ According to Chieng et al., ${ }^{12}$ the crystallinity index is the ratio of the crystalline to the amorphous regions of cellulose. Figure 8 shows the XRD patterns of OP, OPDC and MCC.

The untreated OP possessed the lowest percentage of crystallinity, with $23.54 \%$, as it had a high amount of amorphous region. An increase in the crystallinity of OPDC $(80.14 \%)$ indicated the removal of hemicelluloses and lignin in the amorphous phase after the alkaline and bleaching treatments. By comparison, it was clearly observed that the diffraction peak of OPDC became sharper than that of $\mathrm{OP},{ }^{14}$ corresponding to an increase in crystallinity. The presence of cellulose derived from OP was confirmed by the high intensity and sharpness of the peak located at a $2 \theta$ value of $22^{\circ}$, which is the common value of cellulose type I. On the contrary, the amorphous 
phase of cellulose type I was characterized by a low diffracted intensity peak at a $2 \theta$ value of around $18^{\circ} .^{1}$ The crystallinity index calculated for MCC $(87.69 \%)$ was slightly higher than that of OPDC (80.14\%). In addition, the peak of MCC located at a $2 \theta$ value of $22^{\circ}$ was sharper than that of OPDC, which indicated that MCC was more crystalline than OPDC.

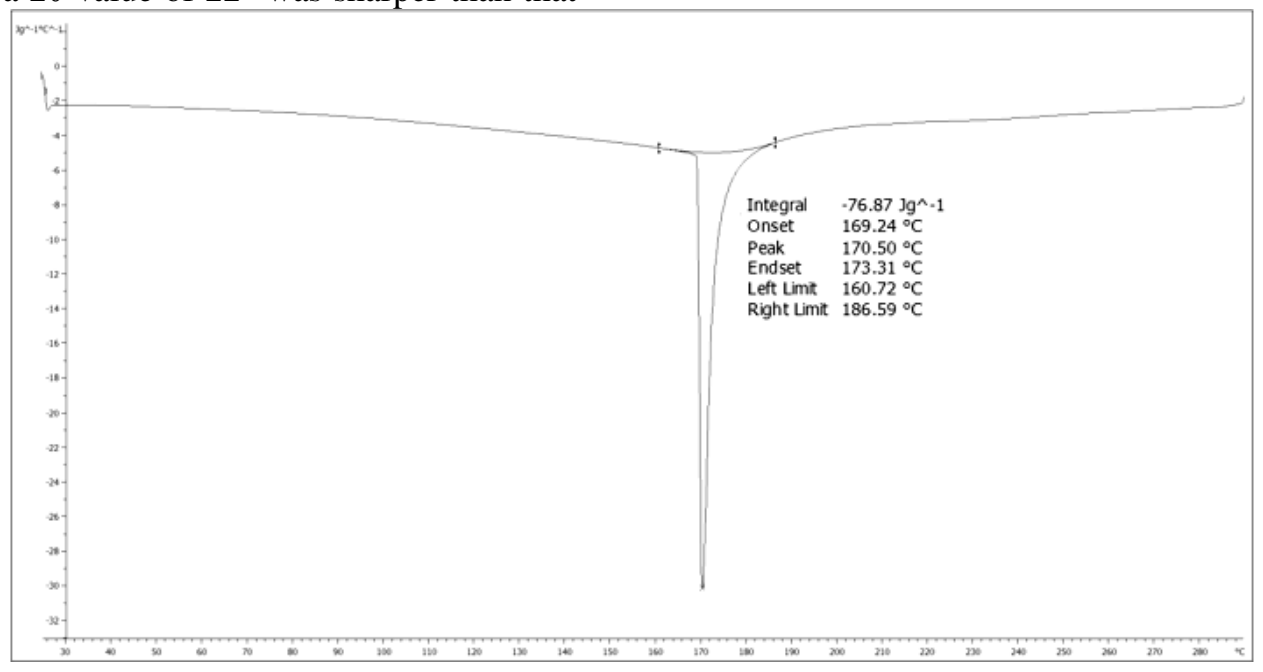

Figure 6: DSC curve of OPDC

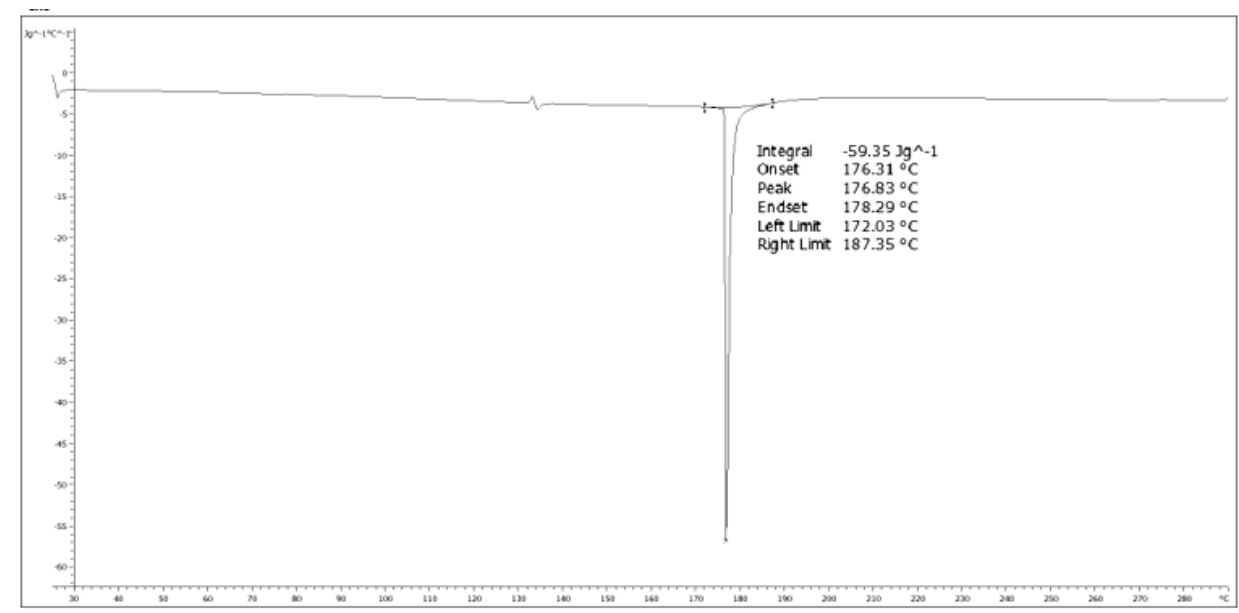

Figure 7: DSC curve of MCC

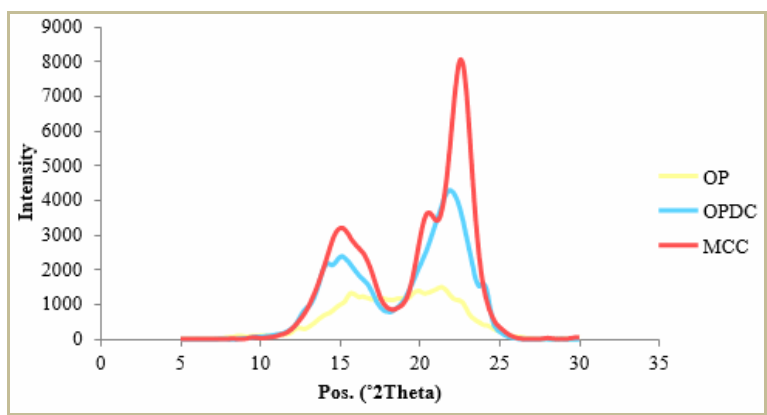

Figure 8: XRD curves of OP, OPDC and MCC 


\section{VANESSA NG WEN YI et al.}

According to Wan, Wang and Xiao, ${ }^{34}$ residual hemicelluloses in $\alpha$-cellulose were found to have a more amorphous structure than the cellulose that had undergone breakdown. Cellulose extraction by alkaline treatment removed the major quantity of lignin, whereas the acid hydrolysis process used to produce MCC eliminated the residual amount of the amorphous lignin.

As reported in the literature, most of the residual hemicelluloses were dissolved and the lignin-hemicelluloses-cellulose interactions were disrupted after the acid hydrolysis. ${ }^{14}$ During the hydrolysis process, sulphuric acid attacked and invaded the amorphous region of cellulose, causing the hydrolytic cleavage of glycosidic bonds and consequently released the individual crystallites. At the same time, the growth and realignment of monocrystals may occur, leading to an increase in the crystallinity of cellulose and giving sharp diffraction peaks, as shown in the XRD pattern of MCC. In general, both OPDC and MCC samples possessed a high peak intensity of $2 \theta$ angle at $22^{\circ}$, which was attributed to the crystalline structure of their cellulose. Also, the presence of a broad peak around $15^{\circ}$ indicated the amorphous arrangement. Hence, cellulose obtained from OP can be considered as containing cellulose type I structure. ${ }^{12}$

\section{FTIR spectroscopy of blended films}

Figure 9 allows comparing the FTIR spectra of the unfilled PVA and blended PVA/OPDC films, while Figure 10 - those of the unfilled PVA and blended PVA/MCC films. It can be observed that the spectra of each of the blended films (PVA/OPDC and PVA/MCC) are similar to the spectra of the unfilled PVA film.

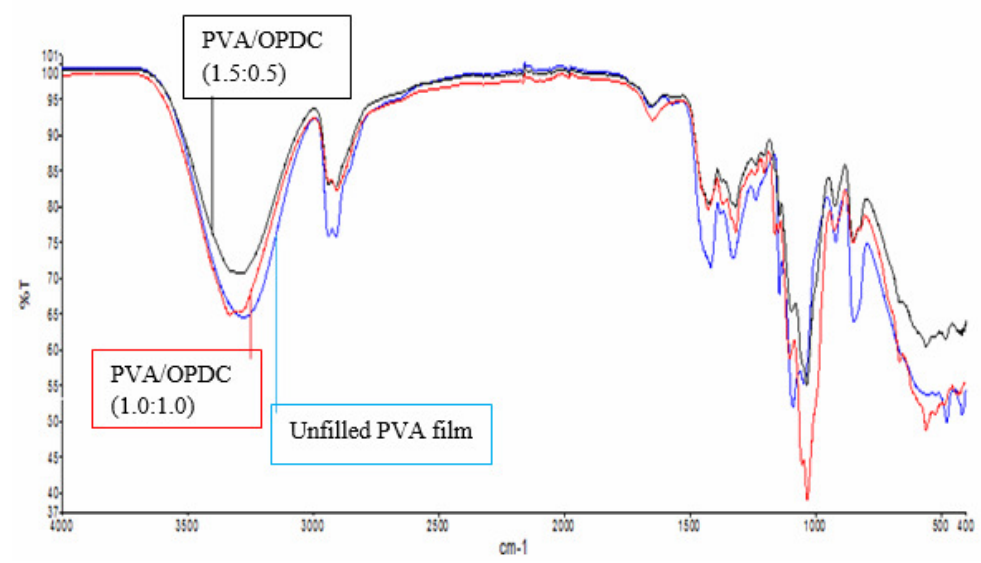

Figure 9: FTIR spectra of unfilled PVA and PVA/OPDC blended films

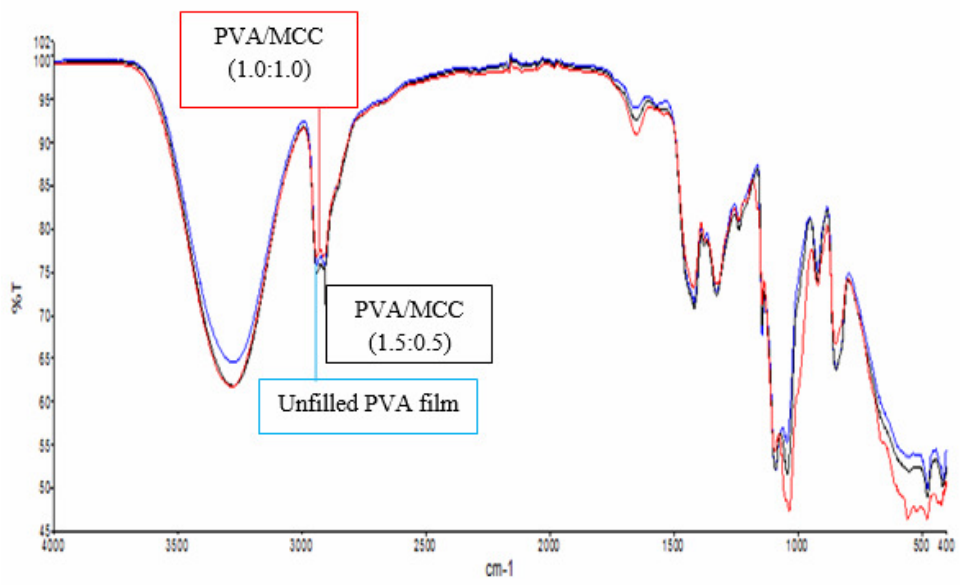

Figure 10: FTIR spectra of unfilled PVA and PVA/MCC blended films 
The shift of the characteristic absorption band and the change in intensity of the band observed in the spectrum of the PVA/OPDC blended film are similar to those of the PVA/MCC blended film. This confirms the development of new interand intra- molecular hydrogen bonds and a change in the conformation between PVA and cellulose. $^{21}$

A band at $3272 \mathrm{~cm}^{-1}$ indicated the $\mathrm{O}-\mathrm{H}$ stretching vibration of the hydroxyl group of PVA. The addition of OPDC into the PVA solution caused a slight decrease in the intensity of $\mathrm{O}-\mathrm{H}$ stretching in the regions around 3272$3289 \mathrm{~cm}^{-1}$ on the spectra of the PVA/OPDC blend films. This can be ascribed to the interface between the hydroxyl groups on the surface of OPDC and the hydroxyl group in PVA. ${ }^{22}$ Similar behaviour was reported by Attia and El-Kader ${ }^{23}$ on PVA/2HEC polyblend films.

However, the PVA/MCC blended films (1.5:0.5 weight ratio) and PVA/MCC blended films (1.0:1.0 weight ratio) showed the peaks at $3272 \mathrm{~cm}^{-1}$ and $3282 \mathrm{~cm}^{-1}$, respectively, with a shift to higher wavenumbers, therefore indicating that there were poor interactions of different $\mathrm{OH}$ groups between PVA and MCC during the process of blending and casting of the solution. ${ }^{24}$ There was no absorption peak detected at 3600 $\mathrm{cm}^{-1}$, indicating the absence of hydroxyl groups present in the unfilled PVA film, and in the PVA/OPDC and PVA/MCC blended films.

According to the literature, the peaks in the regions around 1032-1044 $\mathrm{cm}^{-1}$ were contributed by the $\mathrm{C}-\mathrm{O}-\mathrm{C}$ bending of the pyranose ring in cellulose, ${ }^{17}$ which indicated an increase in intermolecular bonding within the $\mathrm{C}-\mathrm{O}$ in the $\mathrm{C}-$ $\mathrm{O}-\mathrm{C}$ groups. It was also reported that the absorption band shifted to a lower wavenumber, reflecting an increase in intermolecular hydrogen. ${ }^{25}$

Lastly, the absorption peak around $900 \mathrm{~cm}^{-1}$ was assigned to $\mathrm{C}-\mathrm{O}-\mathrm{C}$ stretching of the $\beta$ glycosidic linkages in cellulose. ${ }^{20}$ Siddaiah et al. ${ }^{22}$ reported that the small absorption band around $918 \mathrm{~cm}^{-1}$ was attributed to the characteristic of syndiotactic structure of the films due to the syndiotacticity of PVA, causing dense molecular packing in the crystal and stronger intermolecular hydrogen bonds. This, in turn, led to the restriction of the molecular motion.

As reported previously, the similarity in shape and location of the characteristic peaks of the unfilled PVA film and the blended films (PVA/OPDC and PVA/MCC) suggested that the interactions among PVA molecules were predominant and were stronger than those of PVA-OPDC molecules and PVA-MCC molecules in the blending system. ${ }^{24}$

In addition, the absorption peak at $2909 \mathrm{~cm}^{-1}$ in the spectrum of the unfilled PVA film corresponded to the aliphatic saturated $\mathrm{C}-\mathrm{H}$ stretching. This absorption peak has shifted towards higher stretching frequency observed in the regions around $2910-2913 \mathrm{~cm}^{-1}$ in the blended films. It can also be seen that the intensity of the peaks increased with the increase of PVA content in the blended films. ${ }^{20,22}$ Next, the absorption peaks around $1648-1658 \mathrm{~cm}^{-1}$ were assigned to the $\mathrm{O}-\mathrm{H}$ bending of adsorbed water. ${ }^{26}$ Moreover, the absorption peak at $1417 \mathrm{~cm}^{-1}$ in the spectrum of the unfilled PVA film indicated $\mathrm{CH}_{2}$ symmetric bending vibration. This band is also known as the "crystallinity band". In the spectra of the PVA/OPDC and PVA/MCC blended films, there was a decreased in peak intensity, reflecting a decline in the degree of crystallinity of PVA. ${ }^{27}$

\section{Tensile properties}

Tensile tests were carried out on the PVA/OPDC blended films to determine their tensile strength, elongation at break and elastic modulus, in comparison with the properties of the PVA/MCC blended films. Figures 11-13 show the tensile strength, elongation at break and elastic modulus of the PVA/MCC and PVA/OPDC blended films with different compositions. As shown in Figure 11, the tensile strength of the PVA/MCC and PVA/OPDC blended films decreased from $35 \mathrm{MPa}$ to $3 \mathrm{MPa}$ and $9 \mathrm{MPa}$ with increasing MCC and OPDC contents, respectively. ${ }^{24,31}$

The same trend can also be observed for the elongation at break of the PVA/MCC and PVA/OPDC blended films in Figure 12. It was noticed that the pure PVA film had the highest value of elongation at break - of $377 \%$. However, an addition of $0.5 \%(\mathrm{wt} / \mathrm{wt}) \mathrm{MCC}$ or OPDC during the preparation of blended films caused an adverse effect for the elongation at break, as a sharp decrease was observed from $377 \%$ to $115 \%$ and $37 \%$, respectively. ${ }^{17,36}$

The significant reduction of tensile strength and elongation at break probably indicates weak intermolecular hydrogen bonding between cellulose (MCC and OPDC) and PVA. It was reported that intramolecular hydrogen bonds cause OPDC and MCC to agglomerate, leading to 


\section{VANESSA NG WEN YI et al.}

poor dispersion and poor adhesion ${ }^{11}$ of cellulose in the PVA matrix. ${ }^{10,24}$ Besides, the coalesced and coarsely dispersed OPDC granules acted as stress concentrators, which induced cracks that lowered the values of tensile strength and elongation at break. According to literature, PVA is hydrophilic, while OPDC is semi-hydrophilic due to the presence of oils and fats, which were not completely removed during the pretreatment. OPDC served as filler in the PVA matrix, but as the PVA/OPDC blended films are subjected to the tensile load, only the PVA matrix will mainly withstand the load. This is caused by the less effective cross-sectional area of OPDC at high OPDC content. ${ }^{31}$

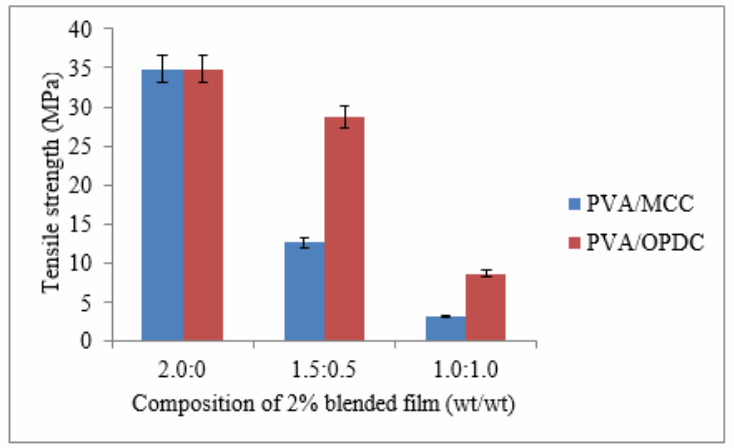

Figure 11: Tensile strength of PVA/MCC and PVA/OPDC blended films with different composition

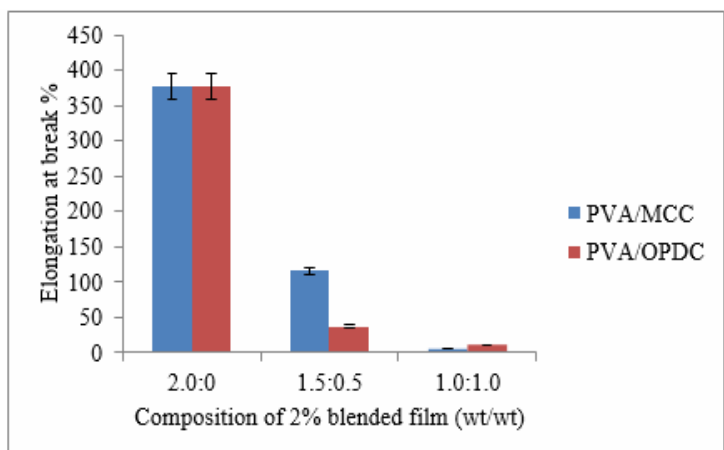

Figure 12: Elongation at break of PVA/MCC and PVA/OPDC blended films with different composition

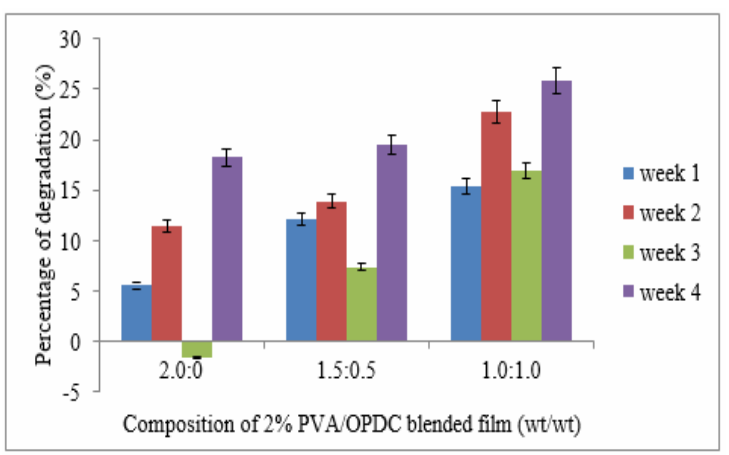

Figure 14: Percentage of degradation of PVA/OPDC blended films with different composition after being exposed to natural weathering for 1 month

The elastic modulus, also known as Young's modulus, of the PVA/MCC blended film increased from $18 \mathrm{MPa}$ to $125.7 \mathrm{MPa}$, whereas

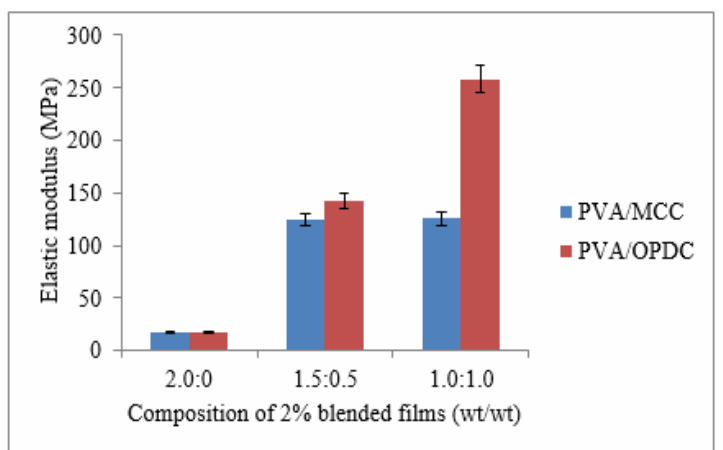

Figure 13: Elastic modulus of PVA/MCC and PVA/OPDC blended films with different composition

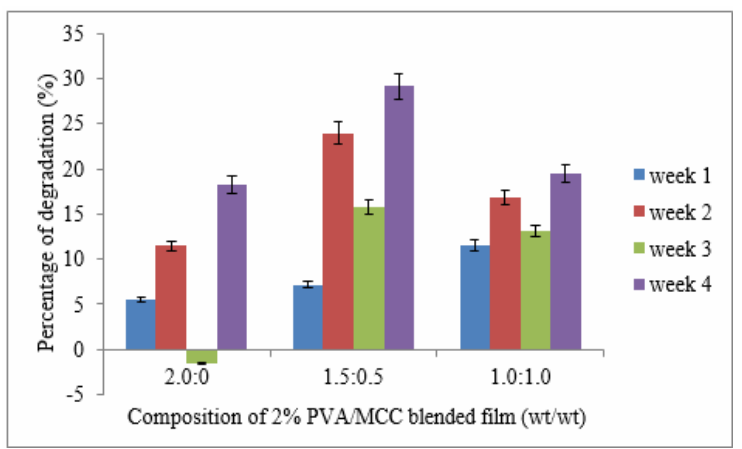

Figure 15: Percentage of degradation of PVA/MCC blended films with different composition after being exposed to natural weathering for 1 month

that of the PVA/OPDC blended film increased from $18 \mathrm{MPa}$ to $258 \mathrm{MPa}$ with increasing MCC and OPDC content, respectively, as shown in 
Figure 13. This was due to the stiffening effect of MCC and OPDC that restricted the molecular mobility of the PVA chains and caused higher resistance of the PVA molecules against straining. Therefore, the presence of MCC and OPDC did not enhance the tensile properties of the blended films because of the poor compatibility of PVA with the celluloses and excellent mechanical properties of PVA itself. ${ }^{31}$

In general, the PVA/OPDC blended films presented mechanical properties very similar to those of the PVA/MCC blended films, with the advantage of higher tensile strength and elastic modulus. Thus, the remarkable mechanical properties of the PVA/OPDC blended film are promising for biotechnological applications. ${ }^{35}$

\section{Natural weathering test}

Figures 14 and 15 show the percentage of degradation of the PVA/OPDC and PVA/MCC blended films with different composition, respectively, after being exposed to natural weathering for 30 days. It was found that all the films were highly deteriorated with decreasing values of weight over time. However, all the films were found to increase in weight on the third week of exposure due to the effect of rainfall, which caused the films to get moistened and wet. However, the water was removed and the films were dried under strong sunlight.

For the unfilled PVA film, the decrease in weight could be attributed to the photooxidation caused by UV exposure and hydrolysis because of
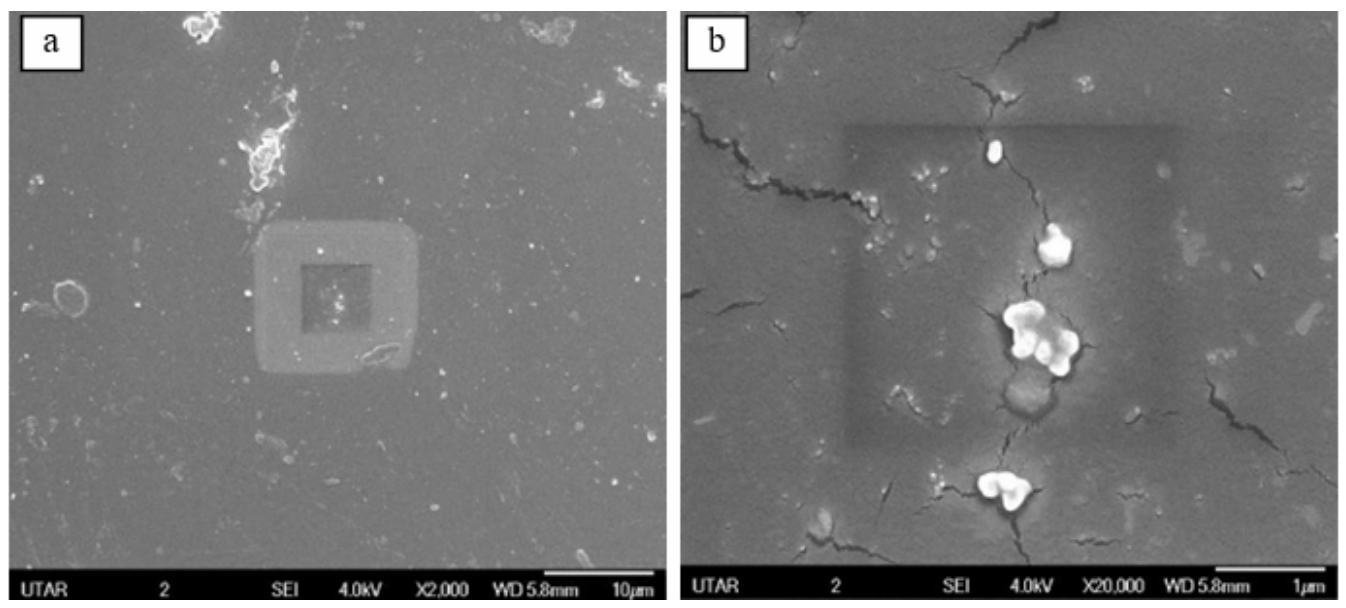

Figure 16: FESEM images of degraded unfilled PVA film after being subjected to natural weathering for 1 rainfall and dew. When raining, PVA tended to absorb water due to the presence of hydroxyl groups, ${ }^{35}$ resulting in a hydrolytic depolymerisation process to convert long polymer chains into smaller oligomer fragments. ${ }^{36}$

According to Figure 14, the percentage of degradation increased with the increasing amount of OPDC. The highest weight loss was exhibited by the PVA/OPDC blended film with the ratio of 1.0:1.0, indicating that the loading of OPDC caused the film to degrade more easily, as compared to the unfilled PVA film. As reported in the literature, cellulose and its derivatives can be easily degraded by microorganisms, such as bacteria, fungi and algae present in air, water and soil. ${ }^{24,35}$ The rate of biodegradation of cellulose depends on the cellulose crystallinity. Since OPDC are less crystalline than dissolved pulp, micro/nanoscale cellulose (in this case MCC), the PVA/OPDC blended film with greater loading of OPDC had a higher biodegradation rate.

According to Figure 15, an insignificant trend of the rate of degradation against the amount of MCC in the film was observed. The highest weight loss was observed for the PVA/MCC blended film with the ratio of 1.5:0.5, whereas the lowest weight loss was shown by the unfilled PVA film. The results indicated that the biodegradability of the PVA/MCC blended film was greatly enhanced by the addition of $0.5 \mathrm{wt} \%$ MCC in the PVA matrix.

\footnotetext{
month with the magnifying power of $(a) \times 2000$ and $(b) \times 20000$
} 


\section{VANESSA NG WEN YI et al.}

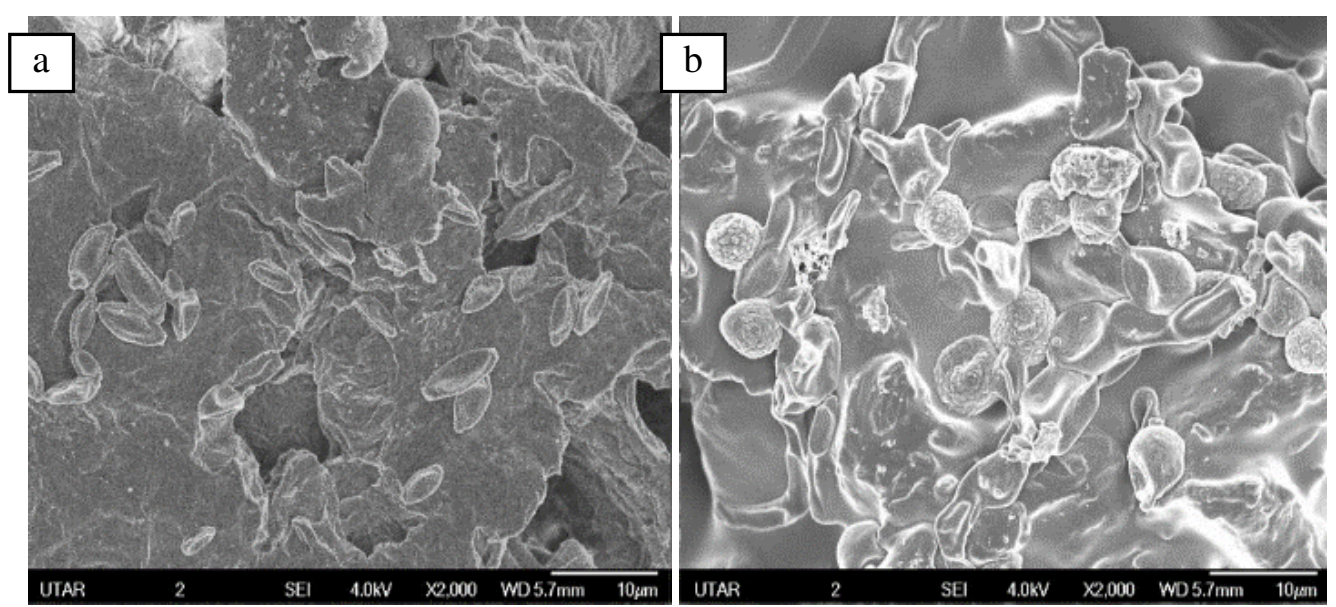

Figure 17: FESEM images of degraded PVA/MCC blended films after being subjected to natural weathering for 1 month with the composition of (a) 1.5:0.5 and (b) 1.0:1.0
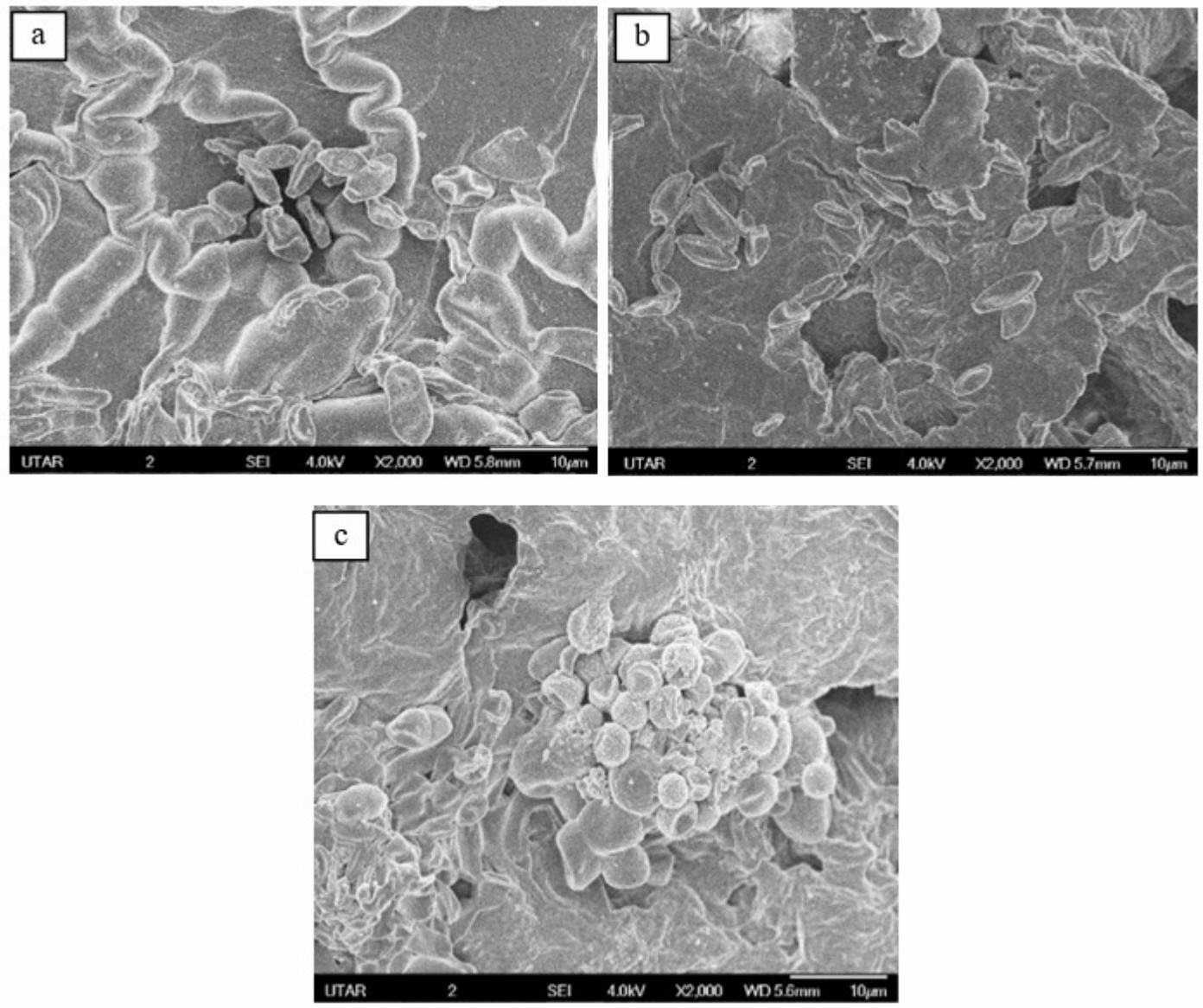

Figure 18: FESEM images of degraded PVA/OPDC blended films after being subjected to natural weathering for 1 month with the composition of (a) 1.5:0.5, (b) 1.0:1.0 and (c) 0.5:1.5

Commercial MCC contained a high amount of cellulose by the elimination of amorphous cellulose regions and other components, such as hemicelluloses and lignin during acid hydrolysis. ${ }^{9}$
Due to the highly ordered arrangement of the cellulose, the film became more compact, causing resistance to the penetration of microorganisms into the film for growth. ${ }^{38}$ This explained the 
reduction in the percentage of degradation as the loading of MCC increased to $1.0 \mathrm{wt} \%$. In the PVA/MCC blended films, the percentage of degradation was lower for the film that contained a lower amount of PVA due to the lack of water solubility, which prevented the diffusion of nutrients into the film to reach the microorganisms. ${ }^{38}$

\section{FESEM of blended films after natural weathering}

Figure 16 shows the FESEM images of the degraded unfilled PVA film after being subjected to natural weathering for 1 month, with different magnifying power. The cracks on the film, as shown in Figure 16 (b), indicate that the film turned brittle. Microbial propagation has been initiated in these cracks and contributed to the degradation of the film. ${ }^{31}$

Figures 17 and 18 show the FESEM images of the degraded PVA/MCC and PVA/OPDC blended films, respectively, after being subjected to natural weathering for 1 month, with different weight ratios. Spherical and rod-like shaped microbes can be seen on the surface of the films. The growth of microbes was actually visible to the naked eye for all the blended films. As compared to the unfilled PVA film, almost the whole surface of both blended films was covered by microbes. This revealed that the addition of OPDC or MCC into the PVA matrix supported the growth of microbes. ${ }^{32}$ It can be concluded that microbes consumed the cellulose, thus creating pores in the PVA film. The morphology studies of the degraded films by FESEM have proven the biodegradability of the blended films due to microbes.

\section{CONCLUSION}

In conclusion, this study demonstrated the feasibility of blending OPDC into a PVA matrix to produce biodegradable PVA/OPDC blended films by the solution casting method. The OPDC was extracted and isolated by alkaline and bleaching treatment from OP, indirectly offering an interesting alternative in waste management of citrus fruit waste. The characterization results, such as FTIR, FESEM and XRD analyses, proved the ability of alkaline and bleaching treatment to remove non-cellulosic components, turning OPDC to micro-size dimensions, with high percentage of crystallinity. However, DSC analysis indicated that MCC possessed higher thermal stability than OPDC.
Apart from that, the development of new interand intra- molecular hydrogen bonds and a change in the conformation between PVA and cellulose was confirmed by the similar trend in the FTIR spectra of the PVA/OPDC and PVA/MCC blended films. An increase in the amount of OPDC and MCC caused a corresponding decrease in the tensile strength and elongation at break of the blended films. However, the elastic modulus of the blended films decreased with a higher amount of OPDC and MCC. The PVA/OPDC blended films presented mechanical properties very similar to those of the PVA/MCC blended films, with the advantage of higher tensile strength and elastic modulus.

The highest weight loss was exhibited by the PVA/OPDC blended film with the ratio of $1.0: 1.0$, proving that the loading of OPDC caused the film to degrade more easily. Tiny black spots on the surface of the film, which reflected the growth of microorganisms, proved the biodegradability of the films. The growth of microorganisms was further proven by the surface morphologies of the degraded films by FESEM. By comparing the PVA/OPDC and the $\mathrm{PVA} / \mathrm{MCC}$ blended films with the same ratio of $1.0: 1.0$, it can be observed that the PVA/OPDC blended film possessed more black spots and higher degradation percentage. This indicated the higher biodegradability of the PVA/OPDC blended film. In short, OPDC proved its potential to serve as cellulose filler in a PVA matrix to produce biodegradable films.

\section{REFERENCES}

1 N. F. M. Zain, S. M. Yusop and I. Ahmad, Nutr. Food Sci., 5, 1 (2014), https://doi.org/10.4172/21559600.1000334

2 H. Chen, "Biotechnology of Lignocellulose", Dordrecht, Springer, 2014, pp. 25-71

3 D. Lavanya, P. K. Kulkarni, M. Dixit, P. Raavi and L. Krishna, International Journal of Drug Formulation and Research, 2, 19 (2011)

4 A. Riley, in "Packaging Technology", edited by A. Emblem and H. Emblem, Woodhead Publishing, 2012, Chapter 14, pp. 310-360, https://doi.org/10.1533/9780857095701.2.310

5 A. Prieto, Microb. Biotechnol., 9, 652 (2016), https://doi.org/10.1111/1751-7915.12393

6 A. Eich, T. Mildenberger, C. Laforsch and M. Weber, PLoS One, 10, p.e0137201 (2015), https://doi.org/10.1371/journal.pone.0137201

7 B. Kord, B. Malekian, H. Yousefi and A. Najafi, Maderas-Cienc. Tecnol., 18, $743 \quad$ (2016), http://dx.doi.org/10.4067/S0718-221X2016005000065 


\section{VANESSA NG WEN YI et al.}

8 Z. X. Ooi, H. Ismail, A. A. Bakar and N. A. A. Aziz, Polym. Plast. Technol. Eng., 50, 705 (2011), https://doi.org/10.1080/03602559.2010.551391

9 B. K. Tan, Y. C. Ching, S. C. Poh, L. C. Abdullah and S. N. Gan, Polymers, 7, 2205 (2015), https://doi.org/10.3390/polym7111509

10 F. M. M. Suki, H. Ismail and Z. A. A. Hamid, Prog. Rubber Plast. Recycl., 30, 103 (2014), https://doi.org/10.1177/147776061403000203

11 J. S. Won, J. E. Lee, D. Y. Jin and S. G. Lee, Int. J. Polym. Sci., 2015, $860617 \quad$ (2015), https://doi.org/10.1155/2015/860617

12 B. Chieng, S. Lee, N. Ibrahim, Y. Then and Y. Loo, Polymers, $\quad 9, \quad 355 \quad$ (2017), https://doi.org/10.3390/polym9080355

13 M. Szymańska-Chargot, M. Chylińska, K. Gdula, A. Kozioł and A. Zdunek, Polymers, 9, 495 (2017), https://doi.org/10.3390/polym9100495

14 M. H. Hussin, N. A. Husin, I. Bello, N. Othman, M.

A. Bakar et al., Int. J. Electrochem. Sci., 13, 3356 (2018), https://doi.org/10.20964/2018.04.06

15 R. A. Ilyas, S. M. Sapuan and M. R. Ishak, Carbohyd. Polym., 181, 1038 (2018), https://doi.org/10.1016/j.carbpol.2017.11.045

16 V. Hospodarova, E. Singovszka and N. Stevulova, Am. J. Anal. Chem., 9, 303 (2018), https://doi.org/10.4236/ajac.2018.96023

17 N. S. Lani, N. Ngadi, A. Johari and M. Jusoh, J. Nanomater., $\quad$ 2014, $13 \quad$ (2014), https://doi.org/10.1155/2014/702538

$18 \mathrm{X}$. Luo and X. Wang, BioResources, 12, 5826 (2017), https://doi.org/10.15376/biores.12.3.5826-5837

19 S. Zarina and I. Ahmad, BioResources, 10, 256 (2014), https://doi.org/10.15376/biores.10.1.256-271

20 M. Fan, D. Dai and B. Huang, in "Fourier Transform-Materials Analysis", edited by S. M. Salih, IntechOpen, 2012, https://doi.org/10.5772/35482

21 M. M. Rahman, S. Afrin and P. Haque, Prog. Biomater., $\quad 3, \quad 23 \quad$ (2014), https://doi.org/10.1007/s40204-014-0023-X

22 T. Siddaiah, P. Ojha, N. O. Kumar and C. Ramu, Mater. Res., 21, e20170987 (2018), https://doi.org/10.1590/1980-5373-mr-2017-0987

${ }_{23}$ G. Attia and M. A. El-Kader, Int. J. Electrochem. Sci., $\quad \mathbf{8}, 5672 \quad$ (2013), http://www.electrochemsci.org/papers/vol8/80405672. pdf

${ }^{24}$ Z. X. Ooi, H. Ismail, A. A. Bakar and N. A. A. Aziz, J. Vinyl Addit. Technol., 17, 198 (2011), https://doi.org/10.1002/vnl.20269

25 Z. X. Ooi, H. Ismail, A. A. Bakar and N. A. A. Aziz, J. Appl. Polym. Sci., 125, 1127 (2012), https://doi.org/10.1002/app.34860

26 M. S. Sarwar, M. B. K. Niazi, Z. Jahan, T. Ahmad and A. Hussain, Carbohyd. Polym., 184, 453 (2018), https://doi.org/10.1016/j.carbpol.2017.12.068

27 D. Ciolacu, F. Ciolacu and V. I. Popa, Cellulose Chem. Technol., 45, 13 (2011), https://cellulosechemtechnol.ro/pdf/CCT12(2011)/p.13-21.pdf

28 Z. N. T. Mzimela, L. Z. Linganiso, N. Revaprasadu and T. E. Motaung, Mater. Res., 21, e20170750 (2018), http://dx.doi.org/10.1590/1980-5373-mr-20170750

${ }^{29}$ H. Chen, Y. Yu, T. Zhong, Y. Wu, Y. Li et al., Cellulose, 24, $333 \quad$ (2017), http://dx.doi.org/10.1007/s10570-016-1116-6

30 A. Kumar, Y. S. Negi, V. Choudhary and N. K. Bhardwaj, J. Mater. Phys. Chem., 2, 1 (2014), http://dx.doi.org/10.1007/978-3-642-27758-0_1162-2

31 R. Shalini and C. Sasikumar, Int. J. Pharma Bio Sci., 6, 447 (2015), retrieved from https://ijpbs.net/abstract.php?article=NDcxNg==

32 F. M. M. Suki, Master's Dissertation, Nibong Tebal, Universiti Sains Malaysia, 2014, http://eprints.usm.my/37236/1/FAIRUS_MAZLIA_BI NTI_MAT_SUKI_24_Pages.pdf

33 R. M. Taib, Z. A. Ghaleb and Z. A. Mohd Ishak, J. Appl. Polym. Sci., 123, $2715 \quad$ (2012), https://doi.org/10.1002/app.34884

34 J. Wan, Y. Wang and Q. Xiao, Bioresour. Technol., 101 4577

(2010),

https://doi.org/10.1016/j.biortech.2010.01.026

${ }^{35}$ F. E. Silva, M. C. B. D. M. Leal, K. D. A. Batista and K. F. Fernandes, J. Mater., 2013, 413578 (2013), https://doi.org/10.1155/2013/413578

36 S. M. Sapuan, H. Ismail and E. S. Zainudin, "Natural Fiber Reinforced Vinyl Ester and Vinyl Polymer Composites: Development, Characterization and Applications", Woodhead Publishing, 2018, https://doi.org/10.1016/B978-0-08-102160-6.00019-6

37 H. P. S. Khalil, Y. Y. Tye, C. K. Saurabh, C. P. Leh, T. K. Lai et al., Express Polym. Lett., 11, 244 (2017), https://doi.org/10.3144/expresspolymlett.2017.26

38 S. Chuayjuljit, S. Su-uthai and S. Charuchinda, Waste Manag. Res., 28, $109 \quad$ (2010), https://doi.org/10.1177/0734242X09339324 